PROCEEDINGS OF THE

AMERICAN MATHEMATICAL SOCIETY

Volume 130, Number 12, Pages 3661-3670

S 0002-9939(02)06630-3

Article electronically published on May 14, 2002

\title{
GLOBAL EXISTENCE AND NONEXISTENCE FOR DEGENERATE PARABOLIC SYSTEMS
}

\author{
YUXIANG LI, WEIBING DENG, AND CHUNHONG XIE
}

(Communicated by David S. Tartakoff)

\begin{abstract}
The initial-boundary value problems are considered for the strongly coupled degenerate parabolic system

$$
\begin{gathered}
u_{t}=v^{p}(\Delta u+a u), \\
v_{t}=u^{q}(\Delta v+b v)
\end{gathered}
$$

in the cylinder $\Omega \times(0, \infty)$, where $\Omega \subset R^{N}$ is bounded and $p, q, a, b$ are positive constants. We are concerned with the global existence and nonexistence of the positive solutions. Denote by $\lambda_{1}$ the first Dirichlet eigenvalue for the Laplacian on $\Omega$. We prove that there exists a global solution iff $\lambda_{1} \geq \min \{a, b\}$.
\end{abstract}

\section{INTRODUCTION}

This paper investigates the global existence and nonexistence of the positive solutions of the strongly coupled degenerate parabolic system

$$
\begin{aligned}
& u_{t}=v^{p}(\Delta u+a u), \\
& v_{t}=u^{q}(\Delta v+b v), \quad \text { in } \Omega_{T} \equiv \Omega \times(0, T)
\end{aligned}
$$

with the first initial-boundary condition

$$
\begin{gathered}
\left.u\right|_{\partial \Omega}=0,\left.\quad v\right|_{\partial \Omega}=0, \\
u(x, 0)=u_{0}(x), \quad v(x, 0)=v_{0}(x), \quad \text { in } \Omega,
\end{gathered}
$$

where $\Omega \subset R^{N}$ is bounded and $p, q, a, b$ are positive constants.

In the past twenty years, the degenerate parabolic equation

$$
\begin{aligned}
& u_{t}=u^{p}(\Delta u+u), \quad \text { in } \Omega_{T}, \\
& \left.u\right|_{\partial \Omega}=0, \\
& u(x, 0)=u_{0}(x), \text { in } \Omega
\end{aligned}
$$

has been studied intensively by many authors. Friedman and McLeod [7 investigated the blow-up property of the positive solutions of (1.3) with $p=2$. They showed that:

(1) If $\lambda_{1}>1$, there exist global nontrivial solutions;

(2) If $\lambda_{1}<1$, no solution is global (if $u_{0} \not \equiv 0$ );

(3) In case (2), the blowup set $S$ has positive measure.

Received by the editors July 23, 2001.

2000 Mathematics Subject Classification. Primary 35K50, 35K55, 35K65.

Key words and phrases. Global existence, global nonexistence, degenerate parabolic system.

(C)2002 American Mathematical Society 
Recently, (1) and (2) have been extended to the problem (1.3) with $p>1$ (see [20]). Moreover, it is shown that

(4) $\lambda_{1}=1$ belongs to the global existence case.

If $p<1$, (1.3) corresponds to the porous media equation. Galaktionov [9] pointed out that this problem has a critical property. Later Pucci and Serrin 17 proved that (1) and (2) hold for its positive solutions. Furthermore, Sacks [18 showed that (4) also holds. Passo and Luckhaus [15] studied (1.3) with $p=1$. They showed that there is no uniqueness of its weak solutions. All these results reflect the statement, emphasized in [12, that large domains $\left(\lambda_{1}<1\right)$ are more unstable than small domains $\left(\lambda_{1} \geq 1\right)$.

Early in the 1980s, Galaktionov et al. [10, [11] studied the porous media system

$$
\begin{aligned}
& u_{t}=\Delta u^{1+\nu}+v^{\alpha}, \\
& v_{t}=\Delta v^{1+\mu}+u^{\beta}, \quad \text { in } \Omega_{T}
\end{aligned}
$$

with the condition (1.2). They proved that $\alpha \beta=(1+\nu)(1+\mu)$ is a critical exponent (see also [12]). But this situation is not as clear as it is for (1.3). However, if $\alpha=1+\mu$ and $\beta=1+\nu,(1)$ and (2) hold. Duan and Zhou [5] studied (1.1), (1.2) of one dimension. It is proved that if $\lambda_{1}>\max \{a, b\}$, then there exists a global positive solution, and if $\lambda_{1}<\min \{a, b\}$, then no positive solution is global and in this case (3) holds.

In this paper we will also go along the thread to investigate the critical exponents of (1.1), (1.2). It might be expected that the critical exponents are combinations of $a$ and $b$. We are particularly concerned with the dependence of the critical exponents upon these parameters.

Throughout this paper, $\partial \Omega \in C^{2+\alpha}$ is assumed to satisfy the outside strong sphere property (see [8, p. 69]) and

$$
\begin{gathered}
u_{0}(x), v_{0}(x) \in C^{1}(\bar{\Omega}), \quad u_{0}(x), v_{0}(x)>0, \quad \text { in } \Omega, \\
u_{0}(x)=v_{0}(x)=0, \quad \partial u_{0} / \partial n, \partial v_{0} / \partial n<0, \text { on } \partial \Omega .
\end{gathered}
$$

Definition 1.1. A positive solution of (1.1), (1.2) is a vector function $(u, v) \in$ $C\left(\overline{\Omega_{T}}\right) \cap C^{2,1}\left(\Omega_{T}\right)$, positive in $\Omega_{T}$ and satisfying (1.1), (1.2).

A crucial role is played here by the first eigenvalue of the Dirichlet problem

$$
\begin{array}{r}
-\Delta \phi(x)=\lambda \phi(x), \quad \text { in } \Omega, \\
\phi(x)=0, \quad \text { on } \partial \Omega .
\end{array}
$$

Denote by $\lambda_{1}$ the first eigenvalue and by $\phi(x)$ the corresponding eigenfunction with the normalization $\phi(x)>0$ in $\Omega$ and $\max _{\bar{\Omega}} \phi(x)=1$. A simple argument with the Rayleigh quotient shows that $\lambda_{1}$ increases as the size of the domain decreases (see [2, Chap. 6]).

Our main result is:

Theorem 1.2. Assume that (H1) holds. Then there exists a global positive solution of (1.1), (1.2) iff $\lambda_{1} \geq \min \{a, b\}$.

To our knowledge, the local existence theory of such a strongly coupled degenerate parabolic system is not established. Since the comparison principle (see [16]) no longer holds generally for the strongly coupled system, we have no monotonicity as to $n$ of the positive solutions $\left(u_{n}, v_{n}\right)$ of the regularized systems (2.2) in Section 2, which is the main difficulty. For the proof of the local existence with monotonicity, 
see [1, 7] and [20]. The outline of proving the local existence is the following. By a modified comparison principle, the positive solutions of the regularized problems can be shown to have uniform upper and lower bounds. Such uniform bounds enable us to have an interior uniform $C^{\alpha}$-estimate (see [13, Theorem 3.1, p. 582]), which can be used to obtain an interior uniform $C^{2+\alpha}$-estimate (see [8, Theorem 5, p. 64]). Now, appealing to the Ascoli-Arzelà theorem, we have a vector function $(u, v) \in C^{2,1}\left(\overline{Q^{\prime}}\right), Q^{\prime} \subset \subset \Omega_{T}$, which satisfies (1.1) in $\overline{Q^{\prime}}$. Next, we extend such obtained $(u, v)$ from $Q^{\prime}$ to $\Omega_{T}$ step by step and $(u, v) \in C^{2,1}\left(\Omega_{T}\right)$ satisfies (1.1) in $\Omega_{T}$. A diagonal procedure implies that there exists a subsequence, also denoted by $\{n\}$, such that $\lim _{n \rightarrow \infty}\left(u_{n}, v_{n}\right)=(u, v)$ in $C^{2,1}\left(\Omega_{T}\right)$. Independently we prove that $(u, v)$ also satisfies the initial-boundary condition (1.2). Thus, $(u, v)$ is a positive solution of (1.1), (1.2).

This paper is organized as follows. Section 2 establishes the local existence theory, Section 3 investigates the global nonexistence and in Section 4 we prove the global existence.

\section{LOCAL EXISTENCE}

In proving the local existence for degenerate parabolic equations, there are three different approaches (see [1, p. 113]). We will modify the boundary conditions rather than the differential equations. Introduce, for $n=1,2, \cdots$, the following regularized system:

$$
\begin{aligned}
& u_{n t}=f_{n}\left(v_{n}\right)\left(\Delta u_{n}+a u_{n}\right), \\
& v_{n t}=g_{n}\left(u_{n}\right)\left(\Delta v_{n}+b v_{n}\right), \quad \text { in } \Omega_{T}, \\
& \left.u_{n}\right|_{\partial \Omega}=1 / n,\left.\quad v_{n}\right|_{\partial \Omega}=1 / n, \\
& u_{n}(x, 0)=u_{0}(x)+1 / n, \quad v_{n}(x, 0)=v_{0}(x)+1 / n, \text { in } \Omega,
\end{aligned}
$$

where

$$
f_{n}\left(v_{n}\right)=\left\{\begin{array}{l}
v_{n}^{p}, \quad v_{n} \geq 1 / n, \\
(1 / n)^{p}, \quad v_{n}<1 / n,
\end{array} g_{n}\left(u_{n}\right)=\left\{\begin{array}{l}
u_{n}^{q}, \quad u_{n} \geq 1 / n, \\
(1 / n)^{q}, \quad u_{n}<1 / n .
\end{array}\right.\right.
$$

By the classical parabolic theory (see [13] or [21]), under (H1), (2.1) has a unique positive solution $\left(u_{n}, v_{n}\right) \in C\left(\overline{\Omega_{T}}\right) \cap C^{2,1}\left(\Omega_{T}\right)$ for $0<T<T_{n}^{*}$, where $T_{n}^{*}$ is the maximal existence time. By a direct computation and the classical maximal principle (see [16, Theorem 13, p. 190]), $u_{n}, v_{n} \geq 1 / n$. Hence, $\left(u_{n}, v_{n}\right)$ satisfies

$$
\begin{aligned}
& u_{n t}=v_{n}^{p}\left(\Delta u_{n}+a u_{n}\right), \\
& v_{n t}=u_{n}^{q}\left(\Delta v_{n}+b v_{n}\right), \quad \text { in } \Omega_{T}
\end{aligned}
$$

with the corresponding initial and boundary condition.

We give two comparison principles frequently used in the following.

Lemma 2.1. Assume that $a_{i}(x, t), b_{i}(x, t), c_{i}(x, t)$ and $d_{i}(x, t)$ are bounded continuous functions and $a_{i}(x, t), b_{i}(x, t) \geq 0, i=1,2$. If a bounded vector function $(w, s) \in C\left(\overline{\Omega_{T}}\right) \cap C^{2,1}\left(\Omega_{T}\right)$ satisfies

$$
\begin{aligned}
& w_{t} \geq a_{1} \Delta w+b_{1} s+c_{1} w+d_{1} w s, \\
& s_{t} \geq a_{2} \Delta s+b_{2} w+c_{2} s+d_{2} w s, \quad \text { in } \Omega_{T}, \\
& \left.w\right|_{\partial \Omega} \geq 0,\left.s\right|_{\partial \Omega} \geq 0, \\
& w(x, 0) \geq 0, \quad s(x, 0) \geq 0, \quad \text { in } \Omega .
\end{aligned}
$$

Then $w, s \geq 0$ in $\Omega_{T}$. 
Proof. Since $(w, s)$ is bounded, a careful examination of the proof of the classical maximum principle (see [16, Theorem 13, p. 190]) shows that this lemma is true in spite of the degeneracy of (2.3).

Proposition 2.2. Assume that $(\bar{u}, \bar{v})$ and $(\underline{u}, \underline{v}) \in C\left(\overline{\Omega_{T}}\right) \cap C^{2,1}\left(\Omega_{T}\right)$ satisfy (1.1) and

$$
\begin{gathered}
\left.\bar{u}\right|_{\partial \Omega} \geq\left.\underline{u}\right|_{\partial \Omega},\left.\quad \bar{v}\right|_{\partial \Omega} \geq\left.\underline{v}\right|_{\partial \Omega}, \\
\bar{u}(x, 0) \geq \underline{u}(x, 0), \quad \bar{v}(x, 0) \geq \underline{v}(x, 0), \quad \text { in } \Omega .
\end{gathered}
$$

If either of them is nondecreasing in $t$, then

$$
(\bar{u}, \bar{v}) \geq(\underline{u}, \underline{v}), \quad \text { in } \Omega_{T} .
$$

Proof. This proposition is a direct consequence of Lemma 2.1

Now we construct a uniform upper bound for $\left(u_{n}, v_{n}\right)$. Consider the system

$$
\begin{aligned}
& \widetilde{u}_{t}=\widetilde{v}^{p}(\Delta \widetilde{u}+a \widetilde{u}), \\
& \widetilde{v}_{t}=\widetilde{u}^{q}(\Delta \widetilde{v}+b \widetilde{v}), \quad \text { in } \Omega_{T}, \\
& \left.\widetilde{u}\right|_{\partial \Omega}=f(t),\left.\quad \widetilde{v}\right|_{\partial \Omega}=g(t), \\
& \widetilde{u}(x, 0)=\theta, \quad \widetilde{v}(x, 0)=\theta, \text { in } \Omega .
\end{aligned}
$$

We choose $\theta=\max _{\bar{\Omega}} u_{0}+\max _{\bar{\Omega}} v_{0}+1$ and assume

$$
\begin{aligned}
& f(t), g(t) \in C^{\infty}([0, \infty)), \quad f^{\prime}(t), g^{\prime}(t)>0, \\
& f(0)=g(0)=\theta, \\
& f^{\prime}(0)=a \theta^{p+1}, g^{\prime}(0)=b \theta^{q+1} .
\end{aligned}
$$

By the classical parabolic theory, under (H2), (2.4) has a unique classical solution $(\widetilde{u}, \widetilde{v}) \in C^{2+\beta, 1+\beta / 2}\left(\overline{\Omega_{T}}\right)$, where $0<\beta<1$ and $0<T<\widetilde{T}^{*}, \widetilde{T}^{*}$ is its maximal existence time. We also have $\widetilde{u}, \widetilde{v} \geq \theta$. From [8. Theorem 12, p. 75], $\left(\widetilde{u}_{t t}, \widetilde{v}_{t t}\right)$ exists in $\Omega_{T}$. Differentiating (2.4) with respect to $t$ and combining with (H2), we obtain a system like (2.3). Lemma 2.1 implies that $\widetilde{u}_{t} \geq 0, \widetilde{v}_{t} \geq 0$ in $\Omega_{T}$. Hence by Proposition 2.2 and the choice of $\theta$, we have

$$
\left(u_{n}, v_{n}\right) \leq(\widetilde{u}, \widetilde{v}), \text { in } \Omega_{T}
$$

for all $n \geq 1$ and $0<T<\widetilde{T}^{*}$. Clearly $T_{n}^{*} \geq \widetilde{T}^{*}$ for all $n$.

Next we give a uniform lower bound. As in [5], let $\widehat{u}=\widehat{v}=k \phi(x) e^{-\rho t}$, where $\phi(x)$ is the first eigenfunction of (1.5), $k>0$ is small such that $u_{0}, v_{0} \geq k \phi(x)$. Fix $T \in\left(0, \widetilde{T}^{*}\right)$. Choose $\rho \geq \lambda_{1} \sup _{\overline{\Omega_{T}}}\left(\widetilde{u}^{q}+\widetilde{v}^{p}\right)$. A direct computation yields that $w=u_{n}-\widehat{u}, s=v_{n}-\widehat{v}$ satisfy

$$
\begin{aligned}
w_{t} & =v_{n}^{p}(\Delta w+a w)+k \phi e^{-\rho t}\left(\rho-\left(\lambda_{1}-a\right) v_{n}^{p}\right) \\
& \geq v_{n}^{p}(\Delta w+a w), \\
s_{t} & \geq u_{n}^{q}(\Delta s+b s), \quad \text { in } \Omega_{T} .
\end{aligned}
$$

Lemma 2.1 implies that

$$
u_{n}, v_{n} \geq k \phi(x) e^{-\rho t}, \quad \text { in } \Omega_{T} .
$$

Combining (2.5) and (2.6), we have for fixed $T \in\left(0, \widetilde{T}^{*}\right)$,

$$
\left(k \phi e^{-\rho t}, k \phi e^{-\rho t}\right) \leq\left(u_{n}, v_{n}\right) \leq(\widetilde{u}, \widetilde{v}), \quad \text { in } \Omega_{T} .
$$


In the following, we employ the so-called extension method outlined in Introduction to prove that there exists a vector function $(u, v) \in C^{2,1}\left(\Omega_{T}\right), 0<T<\widetilde{T}^{*}$, satisfying (1.1) in $\Omega_{T}$. In proving this we need the following regularity. Denote by $c_{i}$ different constants depending on $T$ and $M$, but not on $n$, where $M=\max _{\overline{\Omega_{T}}}(\widetilde{u}+\widetilde{v})$. Denote by $\alpha_{i}$ different constants in $(0,1)$.

Lemma 2.3. $u_{n}, v_{n} \in V_{2}^{1,0}\left(\Omega_{T}\right)$ (see [13] p. 6]).

Proof. We only give the details for $u_{n}$. Multiplying the first differential equation of (2.2) by $u_{n t} / v_{n}^{p}$ and integrating over $\Omega_{t}, 0<t<T$, we get

$$
\begin{aligned}
\int_{\Omega_{t}} \frac{u_{n t}^{2}}{v_{n}^{p}} d x d \tau & =\int_{\Omega_{t}} u_{n t}\left(\Delta u_{n}+a u_{n}\right) d x d \tau \\
& \leq \frac{1}{2} \int_{\Omega}\left(\left|\nabla u_{0}\right|^{2}-\left|\nabla u_{n}\right|^{2}\right) d x+\frac{a}{2} \int_{\Omega} u_{n}^{2} d x .
\end{aligned}
$$

Hence,

$$
\int_{\Omega}\left|\nabla u_{n}\right|^{2} d x \leq c_{1} .
$$

Integrating over $[0, \mathrm{~T}]$ again, we have

$$
\int_{\Omega_{T}}\left|\nabla u_{n}\right|^{2} d x d \tau \leq c_{2}
$$

By the definition of $V_{2}^{1,0}\left(\Omega_{T}\right)$, we have

$$
\left\|u_{n}\right\|_{V_{2}^{1,0}\left(\Omega_{T}\right)}=\max _{0 \leq t \leq T}\left\|u_{n}(., t)\right\|_{2, \Omega}+\left\|\nabla u_{n}\right\|_{2, \Omega_{T}} \leq c_{3} .
$$

This lemma is proved.

Let

$$
\begin{aligned}
& \Omega_{l}=\{x \in \Omega: \operatorname{dist}(x, \partial \Omega)>1 / l\}, \\
& Q_{l}=\Omega_{l} \times(1 / l, T-1 / l) .
\end{aligned}
$$

Without loss of generality, we choose $l=1,2, \cdots$. Hence, we have

$$
\Omega_{l} \subset \subset \Omega_{l+1}, \Omega_{\infty}=\Omega ; \quad Q_{l} \subset \subset Q_{l+1}, Q_{\infty}=\Omega_{T} .
$$

Due to (2.6), we have

$$
u_{n}, v_{n} \geq k \phi(x) e^{-\rho t} \geq \sigma, \quad \text { in } Q_{l} .
$$

Clearly, $\sigma$ depends on $T$ and $l$, but not on $n$. From Lemma 2.3 and applying the interior $C^{\alpha}$-estimate on the compact subset $\bar{Q}_{l+1}$ of $Q_{l+2}$ (see [13, Theorem 3.1, p. 582]) we obtain

$$
\left|u_{n}\right| \frac{\left(\alpha_{1}\right)}{\bar{Q}_{l+1}},\left.\left|v_{n}\right|\right|_{Q_{l+1}} ^{\left(\alpha_{1}\right)} \leq c_{4} .
$$

Also applying the interior $C^{2+\alpha}$-estimate on the compact subset $\bar{Q}_{l}$ of $Q_{l+1}$ (see [8] Theorem 5, p. 64]), we have

$$
\left.\left|u_{n}\right|\right|_{\bar{Q}_{l}} ^{\left(2+\alpha_{2}\right)},\left|v_{n}\right|_{\bar{Q}_{l}}^{\left(2+\alpha_{2}\right)} \leq c_{5} .
$$


In the above procedure let $l=1$, then we have

$$
\left|u_{n}\right|{\frac{\left(2+\alpha_{1}\right)}{Q_{1}}},\left|v_{n}\right|{\overline{Q_{1}}}^{\left(2+\alpha_{3}\right)} \leq c_{6} .
$$

Then the Ascoli-Arzelà theorem implies that there exist a subsequence $\left\{n_{1, k}\right\}$ and a vector function $\left(u^{1}, v^{1}\right) \in C^{2,1}\left(\bar{Q}_{1}\right)$, such that

$$
\lim _{k \rightarrow \infty}\left(u_{n_{1, k}}, v_{n_{1, k}}\right)=\left(u^{1}, v^{1}\right), \text { in } C^{2,1}\left(\bar{Q}_{1}\right)
$$

and $\left(u^{1}, v^{1}\right)$ satisfies (1.1) in $\bar{Q}_{1}$.

Similarly for the case $l=2$, there also exist a subsequence $\left\{n_{2, k}\right\} \subset\left\{n_{1, k}\right\}$ and a vector function $\left(u^{2}, v^{2}\right) \in C^{2,1}\left(\bar{Q}_{2}\right)$, such that

$$
\lim _{k \rightarrow \infty}\left(u_{n_{2, k}}, v_{n_{2, k}}\right)=\left(u^{2}, v^{2}\right), \text { in } C^{2,1}\left(\bar{Q}_{2}\right)
$$

and $\left(u^{2}, v^{2}\right)$ satisfies (1.1) in $\bar{Q}_{2}$.

Since $\left\{n_{2, k}\right\} \subset\left\{n_{1, k}\right\}$, the uniqueness of the limit 2.7) in $\bar{Q}_{1}$ implies

$$
\left(u^{1}, v^{1}\right)=\left.\left(u^{2}, v^{2}\right)\right|_{\bar{Q}_{1}}, \quad \text { in } C^{2,1}\left(\bar{Q}_{1}\right) .
$$

Thus, we extend the vector function $\left(u^{1}, v^{1}\right) \in C^{2,1}\left(\bar{Q}_{1}\right)$ to a vector function $\left(u^{2}, v^{2}\right) \in C^{2,1}\left(\bar{Q}_{2}\right)$ and $\left(u^{2}, v^{2}\right)$ satisfies (1.1) in $\bar{Q}_{2}$. For convenience, we still denote $\left(u^{i}, v^{i}\right)$ by $(u, v)$.

Step by step, we obtain a vector function $(u, v) \in C^{2,1}\left(\Omega_{T}\right)$ satisfying (1.1) in $\Omega_{T}$ and a series of subsequences

$$
\left\{n_{1, k}\right\} \supset\left\{n_{2, k}\right\} \supset \cdots \supset\left\{n_{l, k}\right\} \supset \cdots,
$$

such that

$$
\lim _{k \rightarrow \infty}\left(u_{n_{l, k}}, v_{n_{l, k}}\right)=(u, v) \text {, in } C^{2,1}\left(\bar{Q}_{l}\right) .
$$

By a diagonal procedure, we clearly have a subsequence $\left\{n_{k, k}\right\}$ and

$$
\lim _{k \rightarrow \infty}\left(u_{n_{k, k}}, v_{n_{k, k}}\right)=(u, v), \text { in } C^{2,1}\left(\Omega_{T}\right)
$$

From (2.6), 2.8), we also have

$$
u, v \geq k \phi(x) e^{-\rho t}, \quad \text { in } \Omega_{T} .
$$

Define

$$
\begin{gathered}
\left.u\right|_{\partial \Omega}=0,\left.\quad v\right|_{\partial \Omega}=0, \\
u(x, 0)=u_{0}(x), \quad v(x, 0)=v_{0}(x), \quad \text { in } \Omega .
\end{gathered}
$$

If we can prove $(u, v) \in C\left(\overline{\Omega_{T}}\right)$, combining with (2.8) and (2.9), then $(u, v)$ is a positive solution of (1.1), (1.2).

First we establish the continuity of $(u, v)$ up to $\Omega \times\{0\}$. Note a result of DiBenedetto (see 3, Theorem 1.2, p. 42] or [4]) that if the initial values are only Hölder continuous in an open domain $\Omega^{\prime} \subset \subset \Omega$, then the solution of the Dirichlet problem would be Hölder continuous up to $\Omega^{\prime} \times\{0\}$. Since $u_{0}, v_{0} \in C^{1}(\bar{\Omega}),(u, v)$ is continuous up to $t=0$. [19].

As for the continuity of $(u, v)$ up to $S_{T}$, the proof is standard. We refer to [7] or

Thus far it has been proved that a positive solution of (1.1), (1.2) exists in some interval $(0, T)$. To complete the local existence theory we state a continuation theorem for the positive solution $(u, v)$ defined by (2.8), (2.10). 
Theorem 2.4. Assume (H1) holds and let $T^{*}$ be the supremum over $T$ for which $(u, v)$ exists on $(0, T)$. Then there exists a positive solution of (1.1), (1.2) on $\left(0, T^{*}\right)$. If $T^{*}<\infty$, then $(u, v)$ is unbounded in $\Omega_{T^{*}}$.

Proof. The proof is similar to [6, Theorem 2.5].

\section{The Global NONEXISTEnCE}

In this section we assume

$$
\lambda_{1}<\min \{a, b\} \text {. }
$$

Let $(u, v)$ be any positive solution of (1.1), (1.2). We will prove that, under assumption (3.1), $(u, v)$ does not exist globally (but not means blowup in $C$-norm). First we give a lower bound of the positive solution. The lower bound is of the form $(k \phi(x), k \phi(x))$, where $\phi(x)$ is the first eigenfunction of (1.5), $k>0$ is small such that

$$
u_{0}(x) \geq k \phi(x), \quad v_{0}(x) \geq k \phi(x) .
$$

Denote by $T^{*}$ the maximal existence time of $(u, v)$.

Lemma 3.1. If

$$
\lambda_{1} \leq \min \{a, b\}
$$

then for all $0<T<T^{*}$,

$$
(u, v) \geq(k \phi(x), k \phi(x)), \quad \text { in } \Omega_{T} .
$$

Proof. Let $w=u-k \phi, s=v-k \phi$, then

$$
\begin{aligned}
w_{t} & =v^{p}(\Delta w+a w)+k v^{p}\left(a-\lambda_{1}\right) \phi \\
& \geq v^{p}(\Delta w+a w), \\
s_{t} & =u^{q}(\Delta s+b s)+k u^{q}\left(b-\lambda_{1}\right) \phi \\
& \geq u^{q}(\Delta s+b s) .
\end{aligned}
$$

Since $(u, v)$ is bounded before its maximal existence time $T^{*}$, we have (3.3) according to (3.2) and Lemma 2.1.

Theorem 3.2. If $\lambda_{1}<\min \{a, b\}$, then there are no nontrivial global positive solutions of (1.1), (1.2).

Proof. From (3.1) there exists an open domain $\Omega^{\prime} \subset \subset \Omega$ such that $\lambda_{1}<\lambda_{1}^{\prime}<$ $\min \{a, b\}$, where $\lambda_{1}^{\prime}$ is the first eigenvalue on $\Omega^{\prime}$ of 1.5$)$. Lemma 3.1 implies that

$$
u \geq k \phi(x) \geq 2 \sigma, \quad v \geq k \phi(x) \geq 2 \sigma, \quad \text { in } \Omega_{T}^{\prime}
$$

for some small $\sigma>0$. Then $u_{1}=\left.u\right|_{\Omega^{\prime}}, v_{1}=\left.v\right|_{\Omega^{\prime}}$ satisfy (1.1) in $\Omega_{T}^{\prime}$ with the initial-boundary condition

$$
\begin{gathered}
\left.u_{1}\right|_{\partial \Omega^{\prime}}=\left.u\right|_{\partial \Omega^{\prime}} \geq 2 \sigma,\left.\quad v_{1}\right|_{\partial \Omega^{\prime}}=\left.v\right|_{\partial \Omega^{\prime}} \geq 2 \sigma, \\
u_{1}(x, 0)=u_{0}(x) \geq 2 \sigma, v_{1}(x, 0)=v_{0}(x) \geq 2 \sigma, \quad \text { in } \Omega^{\prime} .
\end{gathered}
$$

Step 1. Consider the following system:

$$
\begin{aligned}
& \underline{u}_{t}=\underline{v}^{p}(\Delta \underline{u}+a \underline{u}), \\
& \underline{v}_{t}=\underline{u}^{q}(\Delta \underline{v}+b \underline{v}), \quad \text { in } \Omega_{T}^{\prime}, \\
& \left.\underline{u}\right|_{\partial \Omega}=f(t),\left.\underline{v}\right|_{\partial \Omega}=g(t), \\
& \underline{u}(x, 0)=\underline{v}(x, 0)=\sigma, \quad \text { in } \Omega^{\prime},
\end{aligned}
$$


where $f(t), g(t)$ satisfy

$$
\begin{aligned}
& f(t), g(t) \in C^{\infty}([0, \infty)), \quad f^{\prime}(t), g^{\prime}(t)>0, \\
& f(0)=g(0)=\sigma, \quad f(t), g(t) \leq 2 \sigma \\
& f^{\prime}(0)=a \sigma^{p+1}, \quad g^{\prime}(0)=b \sigma^{q+1} .
\end{aligned}
$$

By the classical parabolic theory, under (H3), there exists a unique classical solution $(\underline{u}, \underline{v}) \in C^{2+\beta, 1+\beta / 2}\left(\overline{\Omega_{T}^{\prime}}\right)$ to (⒊4) in $\Omega_{T}^{\prime}$ and

$$
\underline{u}, \underline{v} \geq \sigma, \quad \text { in } \Omega_{T}^{\prime} \text {. }
$$

Similarly, by Lemma 2.1, we also have $\underline{u}_{t}, \underline{v}_{t} \geq 0$ in $\Omega_{T}^{\prime}$ and

$$
\Delta \underline{u}+a \underline{u} \geq 0, \quad \Delta \underline{v}+b \underline{v} \geq 0, \quad \text { in } \Omega_{T}^{\prime} .
$$

Thus from Proposition 2.2, we have

$$
(\underline{u}, \underline{v}) \leq\left(u_{1}, v_{1}\right), \quad \text { in } \Omega_{T}^{\prime} .
$$

Therefore, it suffices to show that $(\underline{u}, \underline{v})$ blows up in finite time, because if so, its upper bound $\left(u_{1}, v_{1}\right)$ does exist up to a finite time $T^{*}$.

By (3.5) and (3.6), we have

$$
\begin{aligned}
& \underline{u}_{t} \geq \sigma^{p-\alpha} \underline{v}^{\alpha}(\Delta \underline{u}+a \underline{u}), \\
& \underline{v}_{t} \geq \sigma^{q-\alpha} \underline{u}^{\alpha}(\Delta \underline{v}+b \underline{v}), \text { in } \Omega_{T}^{\prime}
\end{aligned}
$$

with the corresponding initial and boundary condition and $0<\alpha<1$.

Step 2. Consider the problem

$$
\begin{aligned}
& z_{t}=A z^{\alpha}(\Delta z+B z), \quad \text { in } \Omega_{T}^{\prime}, \\
& \left.z\right|_{\partial \Omega^{\prime}}=\sigma, \\
& z(x, 0)=\sigma, \quad \text { in } \Omega^{\prime}
\end{aligned}
$$

with $\lambda_{1}^{\prime}<B \leq \min \{a, b\}$. According to [20, Lemma 3.1], $z(x, t)$ blows up in finite time. Since $\sigma$ is a subsolution of (3.8), the usual subsolution argument (see [14. Lemma 4.1, p. 199]) yields that $z_{t} \geq 0$ and hence $\Delta z+B z \geq 0$. In the following we construct a lower bound for $(\underline{u}, \underline{v})$ of the form $(z, z)$. Let $w=\underline{u}-z, s=\underline{v}-z$ and choose $A \leq \min \left\{\sigma^{p-\alpha}, \sigma^{q-\alpha}\right\}$. Then

$$
\begin{aligned}
w_{t} & \geq \sigma^{p-\alpha} \underline{v}^{\alpha}(\Delta w+a w)+\sigma^{p-\alpha} \underline{v}^{\alpha}(\Delta z+a z)-A z^{\alpha}(\Delta z+B z) \\
& \geq \sigma^{p-\alpha} \underline{v}^{\alpha}(\Delta w+a w)+\alpha \sigma^{p-\alpha} \eta^{\alpha-1}(\Delta z+B z) s, \\
s_{t} & \geq \sigma^{q-\alpha} \underline{u}^{\alpha}(\Delta s+b s)+\sigma^{q-\alpha} \underline{u}^{\alpha}(\Delta z+b z)-A z^{\alpha}(\Delta z+B z) \\
& \geq \sigma^{q-\alpha} \underline{u}^{\alpha}(\Delta s+b s)+\alpha \sigma^{q-\alpha} \xi^{\alpha-1}(\Delta z+B z) w,
\end{aligned}
$$

where $\eta$ lies between $\underline{v}$ and $z$, and $\xi$ lies between $\underline{u}$ and $z$. From $(3.5),(3.8)$ and Lemma 2.1, we have

$$
(\underline{u}, \underline{v}) \geq(z, z), \quad \text { in } \Omega_{T}^{\prime} .
$$

Hence $(\underline{u}, \underline{v})$ blows up in finite time.

According to continuation Theorem 2.4 it follows that the positive solution $(u, v)$ defined by (2.8), (2.10) blows up in finite time. 


\section{The GLOBAL EXISTEnCE}

In this section, we assume

$$
\lambda_{1} \geq \min \{a, b\}
$$

We will prove that, under assumption (4.1), the positive solution $(u, v)$ defined by (2.8), (2.10) exists globally. Without loss of generality, assume $a \leq b$. Let $K>0$ be large such that

$$
u_{0}(x), \quad v_{0}(x) \leq K \phi(x),
$$

where $\phi(x)$ is the first eigenfunction of $(1.5)$. Denote by $T^{*}$ its maximal existence time.

Theorem 4.1. If $\lambda_{1} \geq \min \{a, b\}$, then there exists a global positive solution of (1.1), (1.2).

Proof. Suppose $T^{*}<+\infty$. Let $w=K \phi-u$, then from (4.1), we have

$$
\begin{aligned}
w_{t} & =v^{p}(\Delta w+a w)-K\left(a-\lambda_{1}\right) v^{p} \phi \\
& \geq v^{p}(\Delta w+a w) .
\end{aligned}
$$

Hence (4.2) and Lemma 2.1 imply that $u(x, t) \leq K \phi(x)$ for all $0<t<T^{*}$. Therefore, $K \exp \left(b K^{q} t\right)$ is an upper solution of the equation

$$
\begin{aligned}
& v_{t}=u^{p}(\Delta v+b v), \quad \text { in } \Omega_{T}, \\
& \left.v\right|_{\partial \Omega}=0, \\
& v(x, 0)=v_{0}(x), \quad \text { in } \Omega .
\end{aligned}
$$

It follows that $v(x, t) \leq K \exp \left(b K^{q} t\right)$ for all $0<t<T^{*}$ by Lemma 2.1. This contradicts to continuation Theorem 2.4

\section{ACKNOWLEDGEMENT}

We thank Professor Wang Mingxin for his helpful suggestions on this paper.

\section{REFERENCES}

1. J. R. Anderson, Local existence and uniqueness of solutions of degenerate parabolic equations, Comm. Partial Differential Equations 16 (1991), 105-143. MR 92d:35163

2. R. Courant and D. Hilbert, Methods of mathematical physics I, II, Interscience, New York, 1953, 1962. MR 16:426a; MR 25:4216

3. E. DiBenedetto, Degenerate parabolic equations, Springer-Verlag, New York, 1993. MR 94h:35130

4. E. DiBenedetto, Continuity of weak solutions to a general porous media equation, Indiana Univ. Math. J. 32 (1983), 83-118. MR 85c:35010

5. Z. W. Duan and L. Zhou, Global and blow-up solutions for nonlinear degenerate parabolic systems with crosswise-diffusion, J. Math. Anal. Appl. 244 (2000), 263-278. MR 2001b:35181

6. M. S. Floater, Blow-up at the boundary for degenerate semilinear parabolic equations, Arch. Rational Mech. Anal. 114 (1991), 57-77. MR 92m:35139

7. A. Friedman and B. McLeod, Blow-up of solutions of nonlinear degenerate parabolic equations, Arch. Rational Mech. Anal. 96 (1987), 55-80. MR 87j:35051

8. A. Friedman, Partial differential equations of parabolic type, Prentice-Hall, Englewood Cliffs, N. J., 1964. MR 31:6062

9. V. A. Galaktionov, A boundary value problem for the nonlinear parabolic equation $u_{t}=$ $\Delta u^{\sigma+1}+u^{\beta}$, Differential Equations 17 (1981), 836-842. (Russian) MR 82m:35074]

10. V. A. Galaktionov, S. P. Kurdyumov and A. Samarskii, A parabolic system of quasi-linear equations I, Differential Equations 19 (1983), 1558-1571. 
11. V. A. Galaktionov, S. P. Kurdyumov and A. Samarskii, A parabolic system of quasi-linear equations II, Differential Equations 21 (1985), 1049-1062.

12. H. A. Levine, The role of critical exponents in blowup theorems, SIAM Review 32 (1990), 262-288. MR 91j:35135

13. O. A. Lady ženskaja, V. A. Solonnikov and N. N. Ural'ceva, Linear and quasilinear equations of parabolic type, Amer. Math. Soc., Providence, 1967. MR 39:3159h

14. C. V. Pao, Nonlinear parabolic and elliptic equations, Plenum Press, New York, 1992. MR 94c:35002

15. R. Dal Passo and S. Luckhaus, A degenerate diffusion problem not in divergence form, J. Differential Equations 69 (1987), 1-14. MR 88k:35091

16. M. A. Protter and H. F. Weinberger, Maximum principles in differential equations, SpringerVerlag, 1999.

17. P. Pucci and J. Serrin, A general variational identity, Indiana Univ. Math. J. 35 (1986), 681-703. MR 88b:35072

18. P. E. Sacks, Global behavior for a class of nonlinear evolutionary equations, SIAM J. Math. Anal. 16 (1985), 233-250. MR 86f:35031

19. M. Wiegner, Blow-up for solutions of some degenerate parabolic equations, Differential Integral Equations 7 (1994), 1641-1647. MR 95b:35120

20. S. Wang, M. X. Wang and C. H. Xie, A nonlinear degenerate diffusion equation not in divergence form, Z. Angew. Math. Phys. 51 (2000), 149-159. MR 2001a:35101

21. C. H. Xie, T. P. He and G. H. Bai, Existence and invariance for convection, dispersion, reaction systems with pressure, Acta Math. Sci. 12 (1992), 443-456. MR 94i:35100

Department of Mathematics, Nanjing University, Nanjing 210093, People's Republic OF CHINA

E-mail address: lieyuxiang@yahoo.com.cn

Department of Mathematics, Nanjing University, Nanjing 210093, People's Republic OF CHINA

Department of Mathematics, Nanjing University, Nanjing 210093, People's Republic OF CHINA 